\title{
HYDROMAGNETIC NATURAL CONVECTION FROM A HORIZONTAL POROUS ANNULUS WITH HEAT GENERATION OR ABSORPTION
}

\author{
Jabrane Belabid ${ }^{1, *}$ and Soufiane Belhouideg ${ }^{2}$ \\ ${ }^{1}$ Mohammadia School of Engineers, Mohammed V University, Agdal, Rabat, Morocco \\ ${ }^{2}$ Team of Applied Physics and New Technologies, Polydisciplinary Faculty, Sultan Moulay Slimane University, Beni Mellal, Morocco
}

\begin{abstract}
The problem of unsteady laminar, two-dimensional hydromagnetic natural convection heat transfer in a concentric horizontal cylindrical annulus filled with a fluid-saturated porous medium in the presence of a transverse magnetic field and fluid heal generation effects is studied numerically. It is assumed that the inner and outer walls of the cylindrical annulus are maintained at uniform and constant temperatures $\mathrm{Ti}$ and To respectively. The model consists of the heat equation and the equations of motion under the Darcy law. The derived problem with the stream function-temperature formulation is solved numerically using the alternating direction implicit method. This investigation concerns the effect of magnetic field inclination angle, Hartmann number and heat generation on the heat transfer and the flow pattern. The obtained numerical results are presented graphically in terms of streamlines and isotherms. It was found that the heat transfer mechanisms and the flow characteristics depend strongly on the magnetic field inclination angle, Hartmann number and heat generation..
\end{abstract}

\section{NOMENCLATURE}

$C_{p} \quad$ fluid specific heat at constant pressure

$G$ acceleration of the gravity

$I \quad$ electric current

$K \quad$ permeability

$\alpha \quad$ thermal diffusivity

$\beta \quad$ coefficient of thermal expansion

$\Theta$ angle of inclination to the horizontal of applied magnetic field

$\mu \quad$ dynamic viscosity

$\bar{N} \bar{u}$ average Nusselt number

$P \quad$ Pressure

$Q$ heat generation or absorption coefficient

$R$ radius ratio

$R a$ Rayleigh number of the porous medium

$R a_{c}$ critical Rayleigh number

$r, \varphi$ polar coordinates

$T$ temperature

$t$ Time

$u, v$ polar coordinates of the velocity

$\boldsymbol{V}$ velocity vector

$v \quad$ kinematic viscosity

$\rho$ density

$\sigma \quad$ electric conductivity of the fluid

$\psi$ dimensionless stream function

$\Delta T$ temperature difference dimensional variables

\author{
$i \quad$ inner \\ $o$ outer \\ $\max$ maximum
}

\section{INTRODUCTION}

Natural convective heat transfer in fluid-saturated porous media has received considerable attention over the last few decades. This interest is due to its wide range of numerous applications in geophysics and energy-related engineering problems. Such applications include solar collectors, building heating and cooling system, heat exchangers, thermal storage systems, underground transmission lines, to name just a few (see Vafai [1] and Nield and Bejan [2]).

Natural convection in enclosures in which internal heat generation is present is of crucial interest in certain technological applications. Examples are post-accident heat removal in nuclear reactors and geophysical problems associated with the underground storage of nuclear waste, among others (Acharya and Goldstein [3], Ozoe and Maruo [4], Lee and Goldstein [5], Fusegi et al. [6], Venkatachalappa and Subbaraya [7], Shim and Hyun [8] and Hossain and Wilson [9]).

Magnetic field influence on natural convection has great importance in many industrial applications such as crystal growth, metal casting, liquid metal cooling blankets for fusion reactors, MHD generators and pumps. There has been prime interest in studying the

* Corresponding author: belabide@gmail.com 
influence of a magnetic field on the performance of many systems using electrically conducting fluids [1012].

This paper aims to investigate the effect of magnetic field inclination angle, Hartmann number and heat generation or absorption coefficient on the natural convection in a space bounded by two horizontal concentric cylinders filled with a saturated porous medium.

\section{PROBLEM FORMULATION}

The model considered here is a porous layer saturated with an incompressible Newtonian fluid and bounded between two horizontal concentric cylinders of radius $r_{i}$ and $r_{o}$ as shown in Figure 1. Both inner and outer cylinders are kept at uniform and constant temperatures, respectively, $T_{i}$ and $T_{o}$ where $T_{i}>T_{o}$.

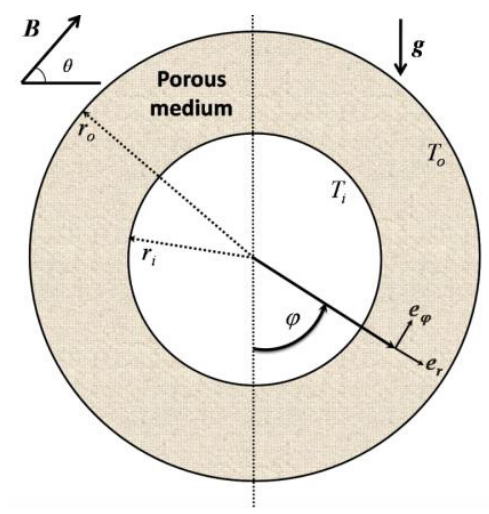

Fig. 1. Physical model geometry.

The fluid is permeated by a uniform external magnetic field, $\boldsymbol{B}$, of constant magnitude $B_{0}$. We assume that the radiation, viscous dissipation, magnetic field induced by the motion of fluid and Joule heating, are taken to be small enough.

The fluid is assumed to be in thermal equilibrium with the porous matrix. The polar coordinates system is used. Under these assumptions along with the Boussinesq approximation, the governing equations of the heat and electric transfer can be written as follows:

$$
\nabla \cdot \boldsymbol{V}^{*}=0
$$

$\boldsymbol{V}^{*}=-\frac{K}{\mu}\left(\nabla P^{*}-\rho \boldsymbol{g}-\boldsymbol{I} \times \boldsymbol{B}\right)$

$$
\begin{aligned}
\frac{\partial T^{*}}{\partial t^{*}}+\boldsymbol{V}^{*} \cdot \nabla T^{*}= & \alpha \nabla^{2} T^{*} \\
& +\frac{Q_{0}}{\rho C_{p}}\left(T^{*}-T_{o}\right)
\end{aligned}
$$

$\nabla . I=0$

$$
\boldsymbol{I}=\sigma(-\nabla \phi+\boldsymbol{V} \times \boldsymbol{B})
$$

where $\boldsymbol{V}^{*}, T^{*}, P^{*}$ and $\boldsymbol{I}$ stand respectively for the dimensional velocity vector, temperature, pressure and the electric current.

$Q_{0}$ is a constant representing the amount of generated or absorbed heat per unit volume from the source. $K$ is the permeability, $\mu$ is the dynamic viscosity, $\rho$ is the density, $\alpha$ is the thermal diffusivity, $t^{*}$ is the time, $g$ is the acceleration of the gravity and $\sigma$ is the electric conductivity and $-\nabla \phi$ is the associated electrical field.

As discussed by Revnic et al. [13] and Garandet et al. [14], equations (4) and (5) reduce to $\nabla^{2} \phi=0$. The unique solution is $\nabla^{2} \phi=0$ since there is always an electrically insulating boundary around the cavity. It follows that the electric field vanishes everywhere (see Alchaar et al. [15]).

It is more suitable to deal with dimensionless equations. Hence, making use of the following transformation parameters: $r=\frac{r^{*}}{r_{i}} ; \quad \boldsymbol{V}=\frac{\boldsymbol{V}^{*}}{\alpha} r_{i} ; T=\frac{T^{*}-T_{O}}{T_{i}-T_{o}} ; t=\frac{t^{*} \alpha}{r_{i}^{2}}$ and $P=\frac{P^{*} K}{\alpha \mu}$, we obtain the following partial differential equations in non-dimensional form:

$$
\begin{aligned}
& \nabla . \boldsymbol{V}=0 \\
& \boldsymbol{V}=-\nabla P-R a T \boldsymbol{k}-H a \boldsymbol{F} \\
& \frac{\partial T}{\partial t}+\boldsymbol{V} \cdot \nabla T=\nabla^{2} T+Q T
\end{aligned}
$$

where $\boldsymbol{k}=\boldsymbol{g} /\|\boldsymbol{g}\|, \quad R a=\frac{g \beta K \Delta T r_{i}}{\alpha \nu}, \quad H a=\frac{\sigma K B_{0}^{2}}{\mu}, \quad Q=$ $\frac{Q_{0} r_{i}^{2}}{\alpha \rho C_{p}}, u$ is the radial component of the dimensionless velocity, $\quad \boldsymbol{F}=\left(u \cos ^{2} \eta+v \sin \eta \cos \eta\right) \boldsymbol{e}_{\boldsymbol{r}}+$ $\left(u \sin \eta \cos \eta+v \sin ^{2} \eta\right) \boldsymbol{e}_{\varphi}$ and $\eta=\theta-\varphi$.

$R a, H a$ and $Q$ are respectively the dimensionless Rayleigh number, Hartmann number and the dimensionless heat generation or absorption parameter.

The governing equations for the laminar natural convection in terms of stream function and temperature are given as:

$$
\begin{gathered}
\nabla^{2} \psi=-R a\left(\sin \varphi \frac{\partial T}{\partial r}+\frac{\cos \varphi}{r} \frac{\partial T}{\partial \varphi}\right) \\
-H a\left(\frac{2 \sin \eta \cos \eta}{r^{2}}\left(\frac{\partial \psi}{\partial \varphi}-r \frac{\partial^{2} \psi}{\partial r \partial \varphi}\right)\right. \\
+\sin ^{2} \eta \frac{\partial^{2} \psi}{\partial^{2} r} \\
\left.+\frac{\cos ^{2} \eta}{r^{2}}\left(\frac{\partial^{2} \psi}{\partial^{2} \varphi}+r \frac{\partial \psi}{\partial r}\right)\right) \\
\frac{\partial T}{\partial t}+\frac{1}{r} \frac{\partial \psi}{\partial \varphi} \frac{\partial T}{\partial r}-\frac{1}{r} \frac{\partial \psi}{\partial r} \frac{\partial T}{\partial \varphi}=\nabla^{2} T+Q T
\end{gathered}
$$


$\psi$ is defined as: $u=\frac{1}{r} \frac{\partial \psi}{\partial \varphi}$ and $v=-\frac{\partial \psi}{\partial r}$. Where $v$ is the tangential component of the dimensionless velocity.

$\beta$ is the coefficient of thermal expansion, $v$ is the kinematic viscosity and $\Delta T=T_{i}-T_{o}$ is the temperature difference. The radius ratio $R=r_{o} / r_{i}$ is another dimensionless parameter of the problem.

The problem is assumed to be symmetric about a vertical line passing through the center of the system, therefore, only one half of the flow domain will be considered. In fact, the boundary conditions can be defined as:

$r=1: T=1$ and $\frac{\partial \psi}{\partial \varphi}=0$

$r=R: T=0$ and $\frac{\partial \psi}{\partial \varphi}=0$.

$\varphi=0, \pi: \frac{\partial T}{\partial \varphi}=0$ and $\frac{\partial \psi}{\partial r}=0$.

The heat transfer across the whole annulus will be presented in terms of the average Nusselt Number evaluated along hot isothermal wall of the annulus as following:

$$
\overline{N u}=-\left.\frac{1}{\pi} \ln R \int_{0}^{\pi} \frac{\partial T}{\partial r}\right|_{r=1} d \varphi .
$$

\section{Numerical methods}

The dimensionless governing equations are discretized using the centered finite difference method coupled with the Alternating Direction Implicit (ADI) scheme. The Thomas Tridiagonal Matrix Algorithm is used in conjunction with iterations to solve the discretized equations. As the study concerns the steady-state regimes, the iterative procedure is stopped when the following relative convergence criterion is satisfied in each node of the grid: $\max \left|\frac{\chi_{i, j}^{\text {new }}-\chi_{i, j}^{\text {old }}}{\chi_{i, j}^{\text {old }}}\right| \leq 10^{-8}$. Where $\chi$ stands for $T$ and $\psi$. The subscripts $i$ and $j$ indices indicate the grid location.

In order to assess the accuracy of the numerical code developed to solve the problem considered in the present investigation, the convection problem was solved within two horizontal concentric cylinders filled with a saturated porous medium in the absence of the magnetic field and the heat generation or absorption effects for which solutions are available. The results obtained using the present code are in good agreement with the data reported earlier by Caltagirone [16] and Alloui and Vasseur [17] as presented in table 1. Moreover, Figure 2 shows a good agreement between the obtained streamlines and temperature contour plots and the results by Charrier-Mojtabi [18], Khanafer et al. [19] and Sheremet and Pop [20] for $R=2$ and $R a=200$.
Table 1. Nusselt number for $R=2$ and $R a=200$. Comparison with literature

\begin{tabular}{|c|c|c|c|}
\hline & $\begin{array}{c}\text { Caltagirone } \\
{[16]}\end{array}$ & $\begin{array}{c}\text { Alloui and } \\
\text { Vasseur [17] }\end{array}$ & $\begin{array}{c}\text { Present } \\
\text { study }\end{array}$ \\
\hline Grid size & $49 \times 49$ & $100 \times 240$ & $49 \times 49$ \\
\hline$R a=50$ & 1.328 & 1.343 & 1.343 \\
\hline$R a=100$ & 1.829 & 1.868 & 1.851 \\
\hline
\end{tabular}

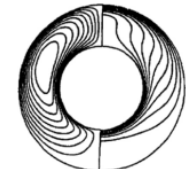

(a)

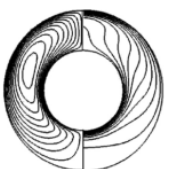

(b)

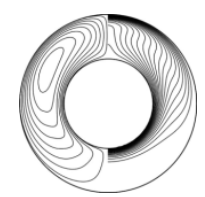

(c)
Fig. 2. Comparison of streamlines and isotherms for $R=2$ and $R a=200$ : numerical results of Charrier-Mojtabi [18] (a), Khanafer et al. [19] (b), Sheremet and Pop [20] (c), present study (d).

For the purpose of obtaining grid independent solution of the problem under consideration, a grid sensitivity analysis is carried out. The present code was tested for grid independence by calculating the average Nusselt number on the hot cylinder. As a matter of fact many numerical experiments were tested for the case of $R=2$, $R a=100, H a=0.5, Q=0.01$ and $\theta=0$. Table 2 shows an effect of the mesh parameters on the average Nusselt number. On the basis of the conducted verifications the grid of $131 \times 131$ was found to yield solutions that are reasonably grid independent.

Table 2. Variations of the average Nusselt number of the hot wall with the grid size.

\begin{tabular}{|c|c|c|}
\hline $\begin{array}{c}\text { Grid size } \\
i \times j\end{array}$ & $\overline{N u}_{i \times j}$ & $\frac{\left|\overline{N u}_{i \times j}-\overline{N u}_{301 \times 301}\right|}{\overline{N u}_{i \times j}} \times 100 \%$ \\
\hline $31 \times 31$ & 1.6239 & 0.0431 \\
\hline $71 \times 71$ & 1.6230 & 0.0123 \\
\hline $101 \times 101$ & 1.6229 & 0.0184 \\
\hline $131 \times 131$ & 1.6232 & 0 \\
\hline $151 \times 151$ & 1.6232 & 0 \\
\hline $201 \times 201$ & 1.6232 & 0 \\
\hline $251 \times 251$ & 1.6232 & 0 \\
\hline
\end{tabular}

\section{Numerical methods}

Numerical investigation of the boundary value problem (7) and (8) has been carried out at the following values of key parameters: Rayleigh number $(50 \leq R a \leq 200)$, Hartmann number $(0 \leq H a \leq 20)$, the Heat absorption or generation parameter $(-0.1 \leq Q \leq 0.1)$, the inclination angle $(0 \leq \theta \leq \pi)$, and a fixed radius ratio $R=2$.

Particular efforts have been focused on the effects of 
these key parameters on the fluid flow and Nusselt number. Figure 3 illustrates the effect of inclination angle on the Nusselt number for $R a=50$. It is interesting to note that the maximum effect of the inclination angle is observed at $\theta=0$.

Figures. 4 and 5 show the Hartmann number on the average Nusselt number for $R a=100$ and different value of the heat generation or absorption coefficient . It is seen that the Nusselt number decreases with increasing the value of $\mathrm{Ha}$ for all values of $Q$ depicted in this investigation. The decrease of $\overline{N u}$ is more significant for $H a<5$.

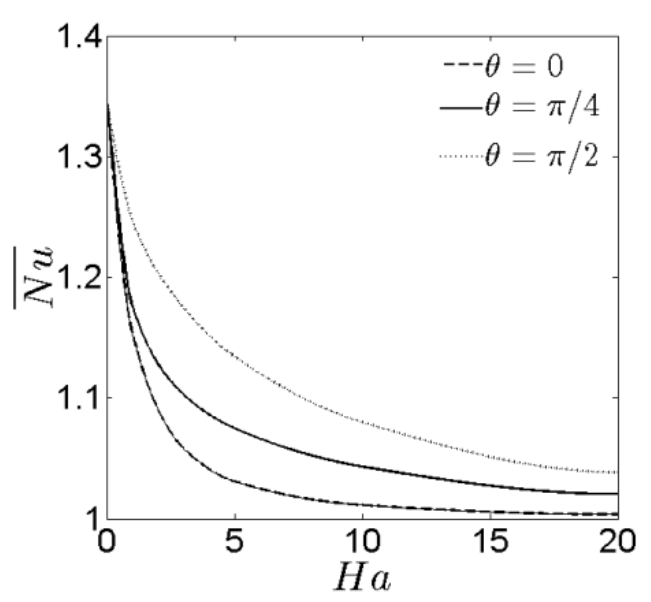

Fig. 3. Average Nusselt number versus Hartmann umber for $R a=50$ and different value of $\theta$.

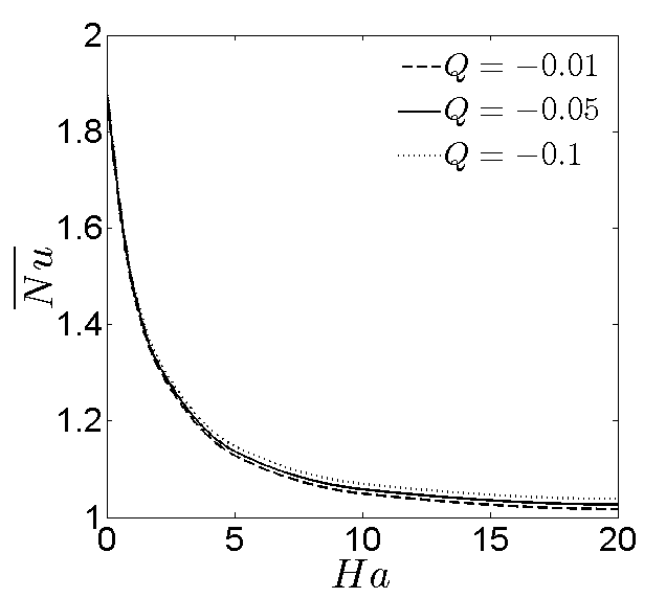

Fig. 4. average Nusselt number versus Hartmann number for $R a=100$ and heat absorption coefficient $(Q<0)$.

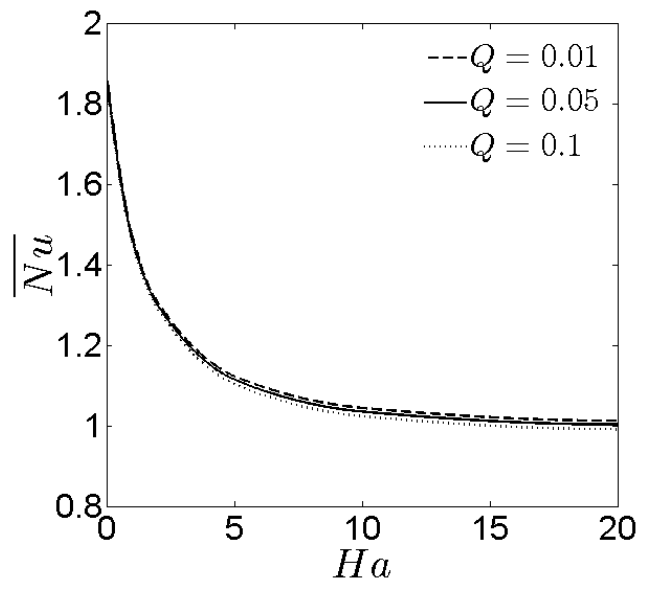

Fig. 5. average Nusselt number versus Hartmann number for $R a=100$ and heat generation coefficient $(Q>0)$.

Figure 6 is plotted to explore the effect of heat generation or absorption coefficient on the average Nusselt number for $\mathrm{Ra}=100$ and different value of Hartmann number It is clear from the figure that as the heat generation or absorption coefficient $Q$ increases, the average Nusselt number decreases.

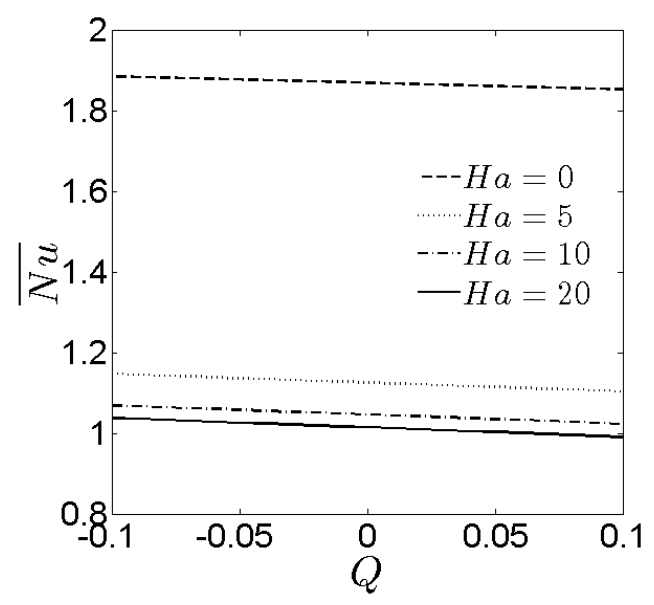

Fig. 6. Average Nusselt number versus $Q$ for $R a=100$ and different values of Hartmann number.

The effect of Hartmann number on the maximum value of the stream function $\psi_{\max }$ is shown in Figure 7 . It is clear from this figure that as $\mathrm{Ha}$ increases, the $\psi_{\max }$ decreases for all values of $Q$. It is noticed also from Figure $7 \mathrm{~b}$ that the maximum value of the stream function increases as the heat generation or absorption coefficient $Q$ increases. 


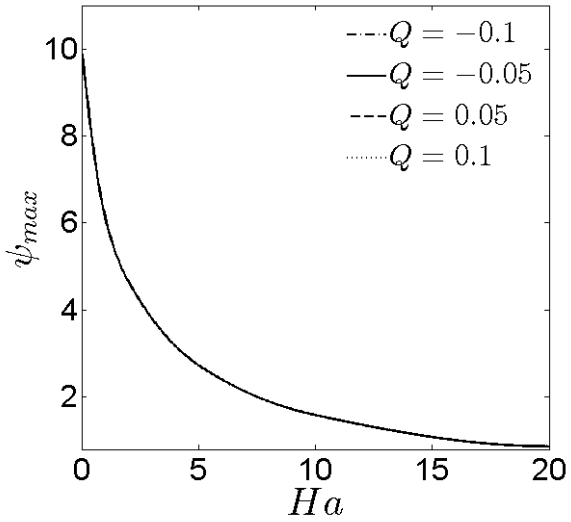

(a)

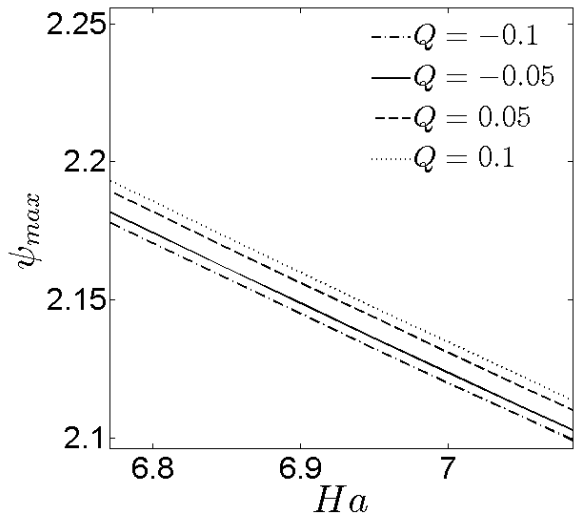

(b)

Fig 7. $\psi_{\max }$ versus $H a$ for $R a=100$ and different values of $Q$.

Figure 8 plots the streamlines and the isotherms for $R a=80, Q=0$ and two values of Hartmann number $H a=0 ; 5$. The flow pattern changes from the bicellular flow structure for $H a=0$ to the unicellular flow structure for $\mathrm{Ha}=5$.

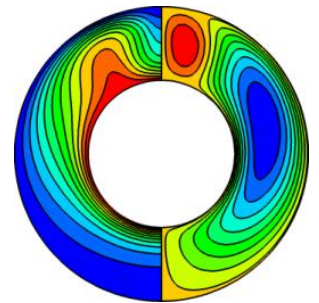

(a)

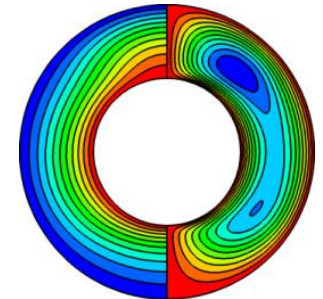

(b)
Fig. 8. Isotherms (left) and streamlines (right) for $R a=80$, $Q=0$ : (a) $H a=0$; (b) $H a=5$.

\section{CONCLUSIONS}

This paper reports a numerical investigation of natural convection heat transfer within a two-dimensional horizontal annulus filled with a saturated porous medium in the presence of a transverse magnetic field and fluid heat generation and absorption effects. The governing equations (written in the stream function and temperature formulation) are solved using the finite difference method with Alternative Direction Implicit scheme. The numerical results are reported for the effect of magnetic field inclination angle, Hartmann number and heat generation and absorption coefficient on the heat transfer and the flow pattern. The obtained numerical results are presented graphically in terms of streamlines and isotherms. It is shown that the heat transfer mechanisms and the flow characteristics depend mainly on the magnetic field inclination angle, Hartmann number and heat generation or absorption coefficient.

\section{References}

1. K. Vafai, Handbook of Porous Media (Third Edition, CRC Press, 2015).

2. D. A. Nield, A. Bejan, Convection in Porous Media (Fifth Edition, Springer International Publishing, 2017).

3. S. Acharya, R. J. Golstein, J. Heat Transfer, 107, pp. 855-866 (1985).

4. H. Ozoe, K. Okada, Int. J. Heat Mass Transfer, 32, pp.1939-1954 (1989).

5. J. H. Lee, R. J. Goldstein, J. Heat Transfer, 110, pp. 345-349 (1988).

6. T. Fusegi, J.M. Hyun, K. Kuwahara, Numer. Heat Transfer A, 21, pp. 215-229 (1992).

7. M. Venkatachalappa, C. K. Subbaraya, Acta Mech., 96, pp. 13-26 (1993).

8. Y.M. Shim, J.M. Hyun, Int. J. Heat Fluid Flow, 18, pp. 328-333 (1997).

9. M.A. Hossain, M. Wilson, Int. J. Therm. Sci., 41, pp. 447-454 (2002).

10. N.G. Kafoussias, Mech. Res. Commun., 19, pp. 8994 (1992).

11. T.K. A1doss, M.A. Al-Nimr, M.A. Jarrah, B.J AlSha'er, Numer. Heat Transfer, part A, 28, pp. 635645 (1995).

12. A.J. Chamkha, Int. Commun. Heat Mass Transfer, 23, pp. 875-887 (1996).

13. C. Revnic, T. Grosan, I. Pop, D.B. Ingham, Int. J. Heat Mass Transfer, 54, pp. 1734-1742 (2011).

14. J. P. Garandet, T. Albussoiere, R. Moreau, Int. J. Heat Mass Transfer, 35, pp. $741-748$ (1992).

15. S. Alchaar, P. Vasseur, E. Bilgen, J. Heat Transfer, 117, pp. 668-673 (1995).

16. J.P. Caltagirone, J. Fluid Mech., 76, pp. 337-362 (1976).

17. Z. Alloui, P. Vasseur, Comp Ther. Sci., 3, pp. 407417 (2011).

18. M. C. Charrier-Mojtabi, Int. J. Heat Mass Transfer, 40, pp. 1521-1533 (1997).

19. K. Khanafer, A. Al-Amiri, I. Pop, Int. J. Heat Mass Transfer, 51, pp 613-1627 (2008).

20. M.A. Sheremet, I. Pop, Computers \& Fluids, 118, pp. 182-190 (2015). 